\title{
Performance Analysis of Rake Receivers in IR-UWB System
}

\author{
Archana \\ EXTC, KJSCE / Mumbai University, India
}

\author{
Swati Mahajan \\ EXTC, KJSCE / Mumbai University, India
}

\begin{abstract}
Suppression of interference in time domain equalizers is attempted for high data rate impulse radio (IR) ultra wideband communication system. The narrow band systems may cause interference with UWB devices as it is having very low transmission power and the large bandwidth. SRAKE receiver improves system performance by equalizing signals from different paths. This enables the use of SRAKE receiver techniques in IR $U W B$ systems A semi analytical approach is used to investigate the BER performance of SRAKE receiver on IEEE 802.15.3a UWB channel models. Study on non-line of sight indoor channel models (both CM3 and CM4) illustrates that bit error rate performance of SRake receiver with NBI performs better than that of Rake receiver without NBI
\end{abstract}

Keywords - Bit error rate, Impulse radio, Non-line of sight, SRAKE receiver, Ultra wideband (UWB)

\section{INTRODUCTION}

UWB technology is based on single band systems employing carrier free or impulse radio communications. Impulse radio (IR) refers to the generation of a series of impulse like waveforms, each of duration in the hundreds of picoseconds. This type of transmission does not require the use of additional carrier modulation and is a baseband signal approach.UWB technology provides high data rate with low power spectral density due to modulation of extremely short pulses within 3.1 to $10.6 \mathrm{GHz}$ [1]. The very low transmission power and the large bandwidth enable an UWB system to co-exist with narrowband communication systems. Although UWB communication offers a promising solution in an increasingly overcrowded frequency spectrum, mutual interference due to coexistence with other spectrally overlapping wireless system degrades the performance of both systems [2]. The interference caused may jam the UWB receiver completely. According to Electromagnetic Compatibility (EMC) reports submitted to FCC, the narrowband interferences (NBI) expected by the UWB receivers are computer motherboard of emission level $42.7 \mathrm{dBm}$ at $1.9 \mathrm{GHz}$, IEEE $802.11 \mathrm{~b}$ at centre frequency $2.4 \mathrm{GHz}$, network interface card (NIC) of emission level $49.8 \mathrm{dBm}$ at $3.75 \mathrm{GHz}$, LAN switch of $44.3 \mathrm{dBm}$ at $3.75 \mathrm{GHz}$, peripheral component interconnect (PCI) card for a personal computer $3.75 \mathrm{GHz}$ and IEEE 802.11a (WLAN system) at centre frequency $5.25 \mathrm{GHz}$ etc. Study of impact of NBI and suppression of NBI is one of the important issues associated with UWB applications. Performance enhancement by employing effective NBI mitigation techniques are discussed in this paper.

Section II introduces the principle of UWB Rake receiver considering its importance in UWB system. In section III UWB Rake receiver structure is analyzed in the presence of NBI. Simulation results for performance analysis of UWB Rake receiver and performance degradation of UWB SRake receiver in presence of NBI are presented.

\section{UWB RAKE RECEIVER STRUCTURE}

The robustness of UWB signals to multipath fading [1] is due to their fine delay resolution, which leads to a high diversity order once combined with a Rake receiver. Rake receivers are used in time-hopping impulse radio systems and direct sequence spread spectrum systems for matched filtering of the received signal. The receiver structure consists of a matched filter that is matched to the transmitted waveform that represents one symbol and a tapped delay line that matches the channel impulse response [3]. It is also possible to implement this structure as a number of correlators that are sampled at the delays related to specific number of multipath components; each of those correlators is known as rake finger. Based upon the Rake receivers are three types. The All-Rake (ARake) receiver captures all most all the energy carried by a very large number of different multipath signals. To reduce the rake complexity, a partial combining (called PRake) is used as partial combining of the energy, which combines the first arriving paths out of the available resolved multipath components. Selective combining 
(called SRake) is a suboptimum Rake receiver, which combines the energy selectively carried out by the strongest multipath components. A UWB Rake receiver structure is shown in Fig.1

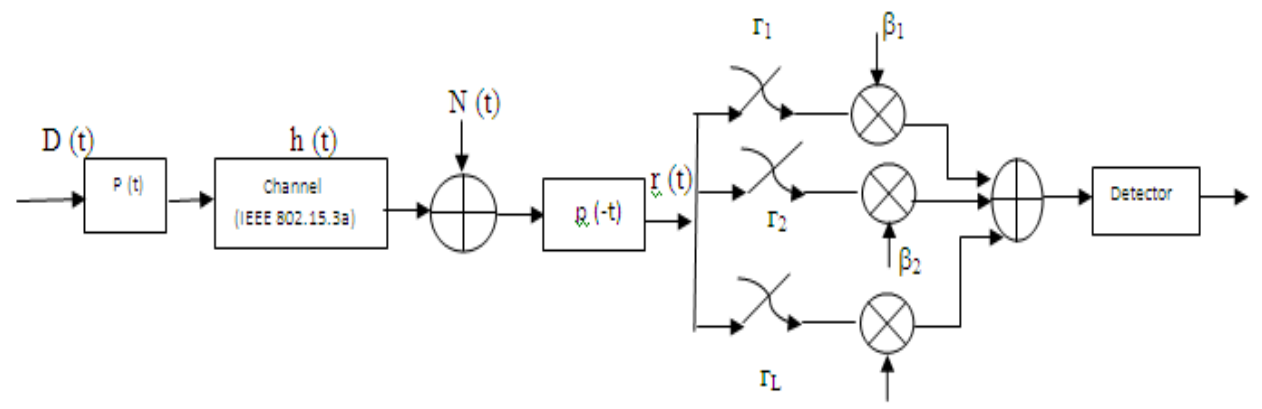

Fig.1 UWB Rake receiver model in presence of NBI

For a single user system, the continuous transmitted data stream is represented as

$$
s(t)=\sum_{k=-\infty}^{+\infty} d(k) p\left(t-k \cdot T_{s}\right)
$$

where $d(k)$ are stationary uncorrelated BPSK data and $T s$ is the symbol duration. The UWB pulse $p(t)$ has duration Tuwb (Tuwb < Ts).

The channel impulse response is given by

$$
h(t)=\sum_{i=0}^{M} h_{i} \delta\left(t-\tau_{i}\right)
$$

$M$ is the total number of paths in the channel

The received signal first passes through the receiver filter matched to the transmitted pulse and is given by:-

$$
\begin{aligned}
r(t) & =s(t) * h(t) * p(-t)+n(t) * p(-t) \\
& =\sum_{k=-\infty}^{+\infty} d(k) \sum_{i} h_{i} m\left(t-k \cdot T_{s}-\tau_{i}\right)+\mathrm{n}(\mathrm{t})
\end{aligned}
$$

Where $p(-t)$ represents the receiver matched filter and $n(t)$ is the Additive White Gaussian Noise (AWGN) with zero mean and variance $N_{o} / 2$. Also, $m(t)=p(t) * p(-t)$ and

$n(t)=n(t) * p(-t)$

$\Lambda$

Combining the channel response with the transmitter pulse shape and the matched filter

$$
\check{h}(t)=p(t) * h(t) * p(-t)=\sum_{i=0}^{M} h_{i} m\left(t-\tau_{i}\right)
$$

The received signal sampled at the $I^{\text {th }}$ rake finger in the $n^{\text {th }}$ data symbol interval is given by

$$
v\left(n . T_{s}+\tau_{l}^{\prime}+t_{0}\right)=\sum_{k=-\infty}^{+\infty} \check{h}\left((n-k) \cdot T_{s}+\tau_{l}+t_{0}\right) \cdot d(k)
$$

Where 
$\tau_{l}^{\prime}$ is the delay time corresponding to the $I^{\text {th }}$ rake finger and is an integer multiple of $\mathrm{T}_{\mathrm{s}}$. Parameter $t_{o}$ corresponds to a time offset and is used to obtain the best sampling time. For the following analysis $t_{o}$ will be set to zero.

The Rake combiner output at time $t=n \cdot T s$ is

$$
y[n]=\sum_{l=1}^{L} \beta_{l} v\left(n \cdot T_{s}+\tau_{l}^{\prime}\right)+\sum_{l=1}^{L} \hat{\beta_{l}} \hat{n}\left(n \cdot T_{s}+\tau_{l}^{\prime}\right)
$$

\section{UWb SRake Receiver Structure In Presence Of Nbi}

The UWB systems must cope with these narrow band interference (NBI) using their high processing gain. However, due to very low transmission power, it is not sufficient to suppress high levels of NBI, which are typically from nearby narrowband radio systems having a bandwidth up to a few $\mathrm{MHz}$ In many cases, the power of NBI is a few tens of dBs higher than both the signal and noise power. The narrowband interference (NBI) signal is modelled as a traditional single carrier BPSK modulated waveform, given by [4]

$$
i(t)=\sqrt{2 P_{l}} \cos \left(w_{0} t+\theta\right) \sum_{p=-\infty}^{\infty} g_{k} z\left(t-k T_{1}-\tau_{1}\right)
$$

where, $P_{l}$ is average transmit power of the narrowband waveform. $\omega_{0}=2 \pi f_{0}$ is carrier frequency of the narrowband waveform. $\theta$ is the random phase of the carrier. $\left\{g_{k}\right\}$ are the randomly modulated BPSK symbols where $\mathrm{g}_{\mathrm{k}} \in\{ \pm 1\}, T_{1}$ is the symbol period, $\tau_{1}$ is a random delay uniformly distributed in $\left[0, T_{l}\right]$ and $z(t)$ is the baseband wave form shape. UWB Rake receiver model considering NBI is shown in Fig. 2

$\{\mathrm{d}(\mathrm{k})\}$

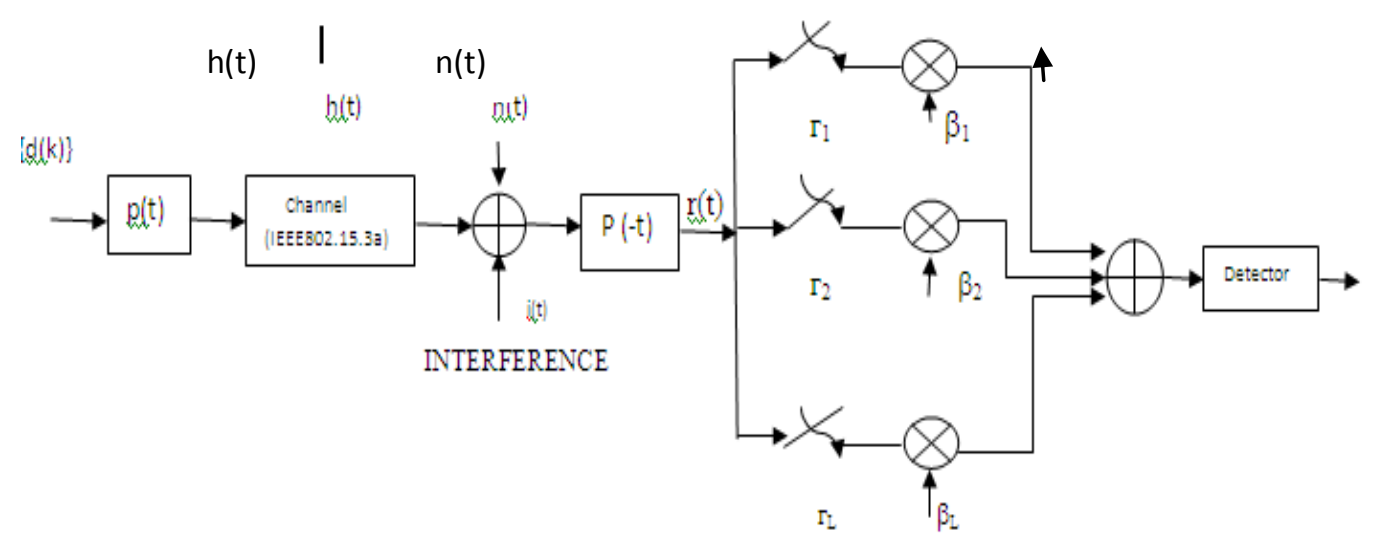

Fig.2 UWB Rake receiver model in presence of NBI

The received signal passes through the receiver filter matched is given by

$$
r(t)=A(t) * h(t) * p(-t)+n(t) * p(-t)+i(t) * p(-t)
$$

Interference coexisting with the same system generates extra signal which can't be easily detected at the output. Rather by coexisting with original pulse, it will decrease the performance of receiver. If such interference is not properly suppressed, then this will jam the receiver and the system performance degrades.

\section{Performance AnAlysis Of Rake Receivers In IR UWB SySTEM}

BER performance of Rake receiver in IR-UWB system is observed through MATLAB simulation [5]. Performance comparison among ARake, SRake, and PRake receiver is carried out using different IEEE UWB channel models as shown in Fig. 3 and 4. Since a UWB signal has a very wide bandwidth, ARake receiver combining all the paths of the incoming signal is practically unfeasible. Therefore, SRake becomes the practical choice. 


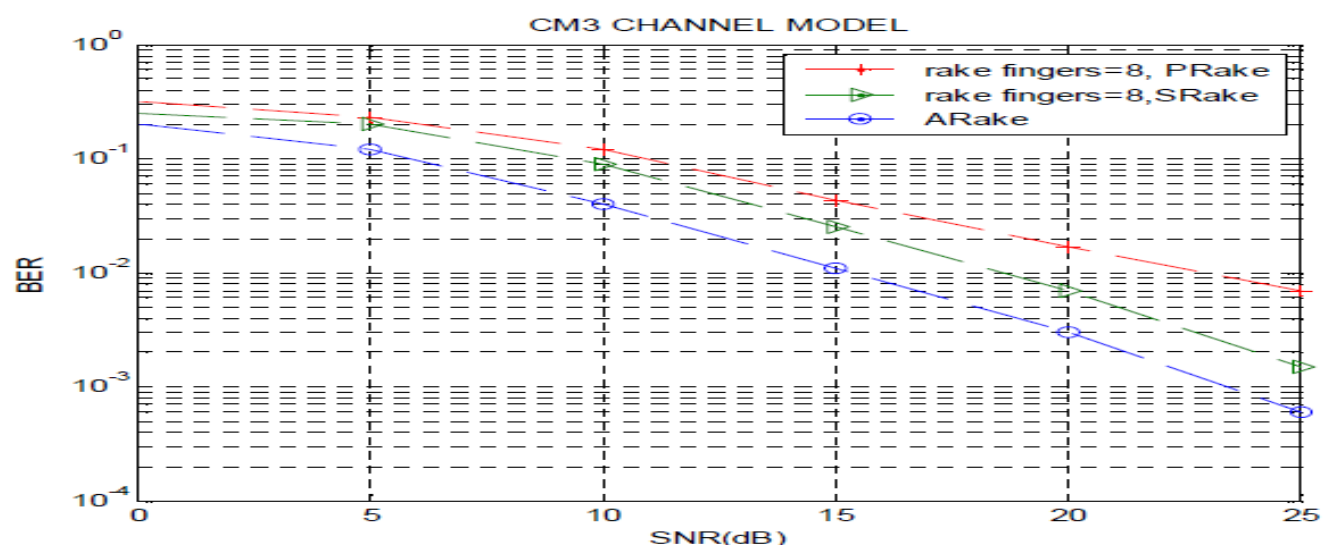

Fig.3 BER performance of Rake receiver for CM3 channel model

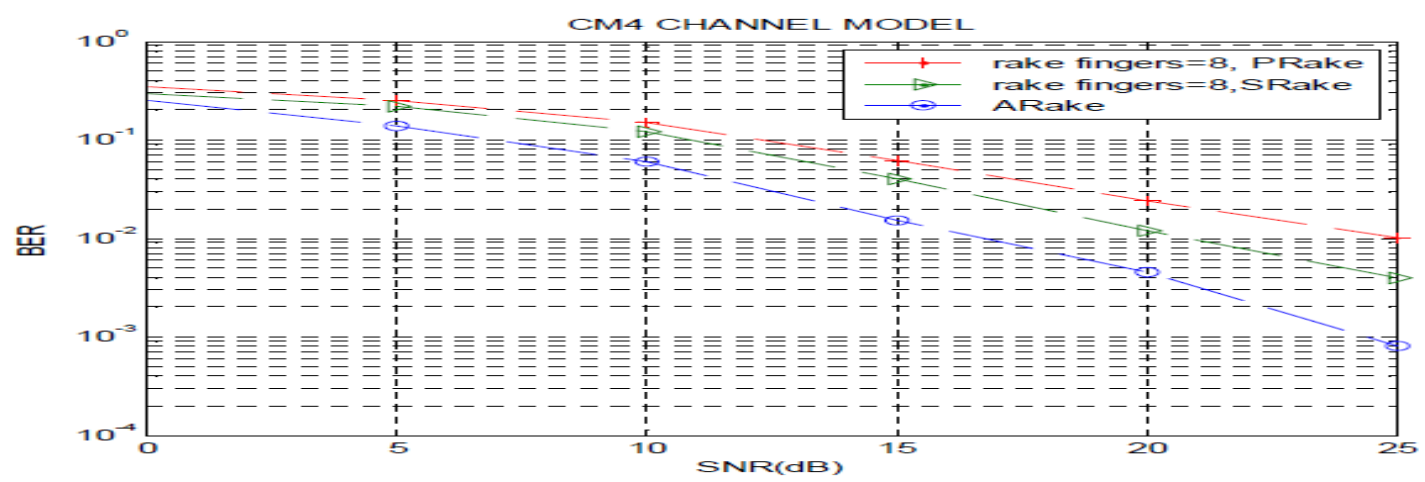

Fig. 4 BER performance of Rake receiver for CM4 channel model

\section{Performance Analysis Of Rake Receivers In Ir Uwb System In Presence Of Nbi}

SRake receiver in UWB system performance in absence and presence of NBI is studied and found that NBI deteriorates the system performance. Simulations are done in Matlab [5].

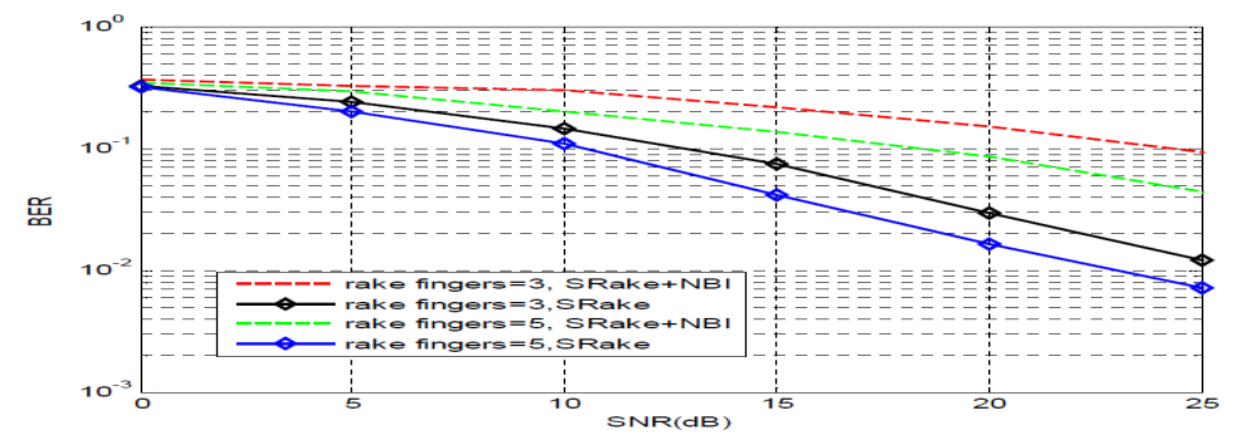

Fig. 5 Performance of SRake receiver for CM3 channel model 


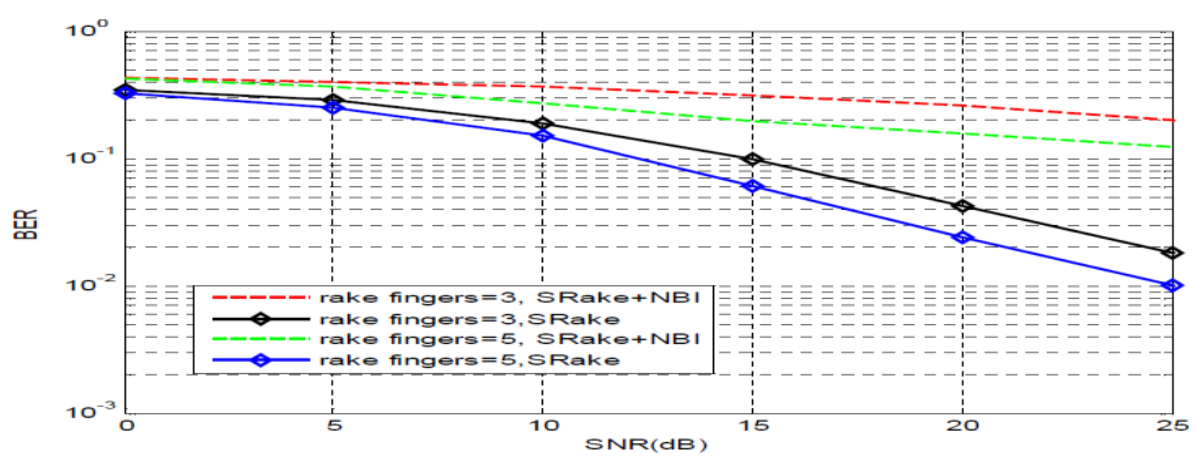

Fig. 6 Performance of SRake Receiver for CM4 channel model

\section{CONCLUSION}

IR-UWB is an emerging as a solution for the IEEE 802.15a (TG3a) standard, which provides low complexity, low cost, low power consumption and high data-rate in Wireless Personal Area Network (WPAN) system. For high data rate and short range, the receiver combats NBI interference by taking advantage of the Rake receiver and SRake structure.

\section{References}

[1] J. R. Foerster, "The Effects of Multipath Interference on the Performance of UWB Systems in an Indoor Wireless Channel," 53rd IEEE VTS, vol.2, no.69, pp.1176- 1180, 2001

[2] L. Zhao, A. M. Haimovich, and H. Grebel, "Performance of ultra-wideband communications in the presence of interference," IEEE proceedings of ICC-01, pp.2948-2952, 2001.

[3] A. Rajeswaran, V. S. Somayazulu, and J. R. Foerster, "Rake performance for a pulse based UWB system in a realistic UWB indoor channel," IEEE International Conference on Communications (ICC'03), vol.4, pp.2879-2883, May 2003.

[4] N. Boubaker and K.B. Letaief, "A Low Complexity RAKE Receiver in a realistic UWB Channel and in the presence of NBI," IEEE Wireless Communications and Networking, (WCNC), vol.1, pp.233-237, 2003

[5] Matlab Version 7.5 